\title{
Optimal Adaptive Filtering Technique for Tamil Speech Enhancement
}

\author{
Vimala.C \\ Project Fellow, \\ Department of Computer Science \\ Avinashilingam Institute for Home Science and \\ Higher Education and Women \\ Coimbatore, India
}

\author{
Radha.V \\ Associate Professor, \\ Department of Computer Science \\ Avinashilingam Institute for Home Science and \\ Higher Education and Women \\ Coimbatore, India
}

\begin{abstract}
Noise reduction in speech applications has a significant amount of research for several decades. The ultimate goal of the speech signal processing research is to develop systems which can perform well in noisy environments. The main objective of this research work is to obtain a clean speech signal of higher quality by applying the optimal noise cancellation technique. In recent years, the real-time adaptive filtering algorithms are considered to be an essential technique for noise cancellation. In this paper, the most popular adaptive filtering algorithms namely Least Mean Square (LMS), Normalized Least Mean Squares (NLMS) and Recursive Least Squares (RLS) algorithms are analyzed and implemented for Tamil speech enhancement. Based on the experimental results, it can be observed that the performance of the adaptive filters is better than using conventional methods designed for speech enhancement. The performances of these algorithms are evaluated based on the metrics namely PSNR, MSE, SNR and SNR Loss. Based on the performance evaluation, the RLS algorithm was found to be a better optimal noise cancellation technique for Tamil speech signals.
\end{abstract}

\section{Keywords}

Tamil Speech, Noise cancellation, adaptive filter, LMS, NLMS, RLS, Speech enhancement, SNR and SNR loss.

\section{INTRODUCTION}

Signal carries of both useful and unwanted information and therefore extracting the valuable information from a mixture of conflicting information is highly needed in any signal processing application. Signal is normally polluted by different kind of noise and therefore extracting the reliable information from the corrupted signal is always important in any signal processing application. The presence of noise in signal generally affects its nature and introduces loss in information and also degrades the performance of the speech recognition system. Hence, the signal has to be cleaned up with noise cancellation technique before it is stored, analyzed, transmitted, or processed [1] [2]. The foremost task in any signal processing application is to offer the optimal input signal for the system. In recent years, with the wide development of Digital Signal Processing (DSP) tools, adaptive filtering techniques have become standard solutions for this issue [3]. The main aim of this research work is to study and implement various adaptive filtering algorithms for Tamil speech enhancement. The well known and popular kind of adaptive filters namely LMS, NLMS and RLS algorithms are implemented and their performances are analyzed both subjectively and objectively to find out the optimal technique for Tamil speech enhancement.

The paper is organized as follows. Section 2 presents the need for speech enhancement, section 3 explains the concept of adaptive filtering and the adopted algorithms for this research work, section 4 investigates the experimental results and section 5 deals with the performance evaluation of the above work. Finally, the conclusion is summarized in section 6 .

\section{NEED FOR SPEECH ENHANCEMENT}

Speech enhancement is one of the most important topics in speech signal processing to improve the performance of the systems in noisy conditions. Speech enhancement is employed in many applications like front-ends for speech recognition systems, enhancement of signals in telecommunications, military, teleconferencing, and cellular environments etc [4]. Noise and distortion are the main limiting factors in communication systems. Therefore, the modeling and removal of the effects of noise and distortion have been at the core of the theory and practice of communications and signal processing [3] [7]. Several techniques have been proposed for this purpose namely spectral subtraction, extended and iterative wiener filtering, adaptive filtering, kalman filtering, fuzzy algorithms, HMM-based algorithms, signal subspace approach and kernel based approaches etc. The performances of these techniques depend on the quality and intelligibility of the processed speech signal. The improvement of the speech signal-to noise ratio is the target of most of the techniques. The following figure 1 shows the basic idea of speech enhancement. 


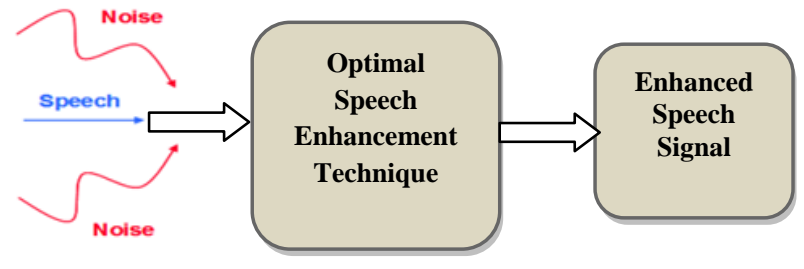

Figure 1: Basic idea of speech enhancement

\section{SPEECH ENHANCEMENT USING ADAPTIVE FILTERS}

Speech noise cancellation techniques are broadly divided into two types namely adaptive and non-adaptive. In general, adaptive filter techniques are superior to non-adaptive filters. Non-adaptive filters cannot process time-varying non stationary signals and they require a priori knowledge of the statistics of the signal to be processed. In case of adaptive filters, it does not require a priori knowledge of signal or noise characteristics. Such a filter with adjustable parameters is called adaptive filter. They are also called self learning filters which make the system to perform adequately in an environment where the relevant information is not presented [9].

Adaptive filters are classified into two main groups namely linear and non linear. In Linear adaptive filters, the computation is done by estimating the desired response with the combination of the available set of observables applied to the input of the filter. Otherwise, the adaptive filter is said to be nonlinear [9]. Adaptive filters are effectively used in a range of applications like channel equalization, system identification, predictive deconvolution, spectral analysis, signal detection, noise cancellation, and beam forming etc. One of the most common practical applications of adaptive filters is noise cancellation.

In adaptive noise cancellation algorithm, the corrupted signal is processed through a filter in order to suppress the noise without losing the speech content. The following figure 2 shows the architecture of adaptive filtering technique applied for speech noise cancellation [8].

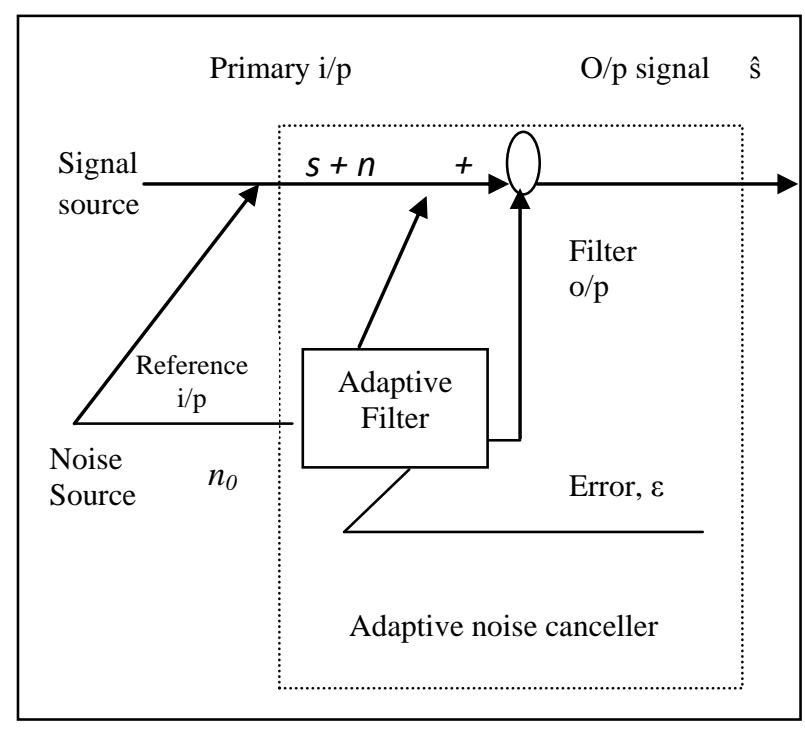

Figure 2: Architecture of Adaptive filtering for speech noise cancellation
As shown in Figure 2, an adaptive filter is a digital filter with coefficients that are determined and updated by an adaptive algorithm. Therefore, the adaptive algorithm behaves like a human operator that has the ability to adapt in a changing environment. A typical performance criterion is based on an error signal, which is the difference between the filter output signal and a given reference (or desired) signal. Based on the metrics of the adaptive filters, the three widely used algorithms are applied to the noisy signals for enhancement and they are explained below.

\subsection{Least Mean Square (LMS) algorithm}

The LMS is the first and mainly used algorithm in adaptive filtering developed by Widrow and Hoff. The LMS algorithm has established itself as the workhorse of adaptive signal processing for three primary reasons:

- Ease of implementation

- Computational efficiency with adjustable parameters

- Robust performance

It is a gradient descent algorithm and it adjusts the adaptive filter taps modifying them by an amount proportional to the instantaneous estimate of the gradient of the error surface [10]. The steps involved in the standard LMS algorithm are given below [11]:

1. Calculates the output signal $y(n)$ from the adaptive filter.

2. Calculates the error signal $e(n)$ by using the following equation (1)

$$
e(n)=d(n)-y(n)
$$

3. Updates the filter coefficients by using the following equation (2)

$$
\varpi(n+1)=\varpi(n)+\mu \cdot e(n) \cdot \bar{u}(n)
$$

Where $\mu$ is the step size of the adaptive filter, $\mathrm{w}$ (n) is the filter coefficients vector, and $\vec{u}(n)$ is the filter input vector [6]. LMS algorithms adjust the filter coefficients to minimize the cost function. They do not involve any matrix operations and therefore it requires fewer computational resources and memory [12].

\subsection{Normalized Least Mean Square (NLMS) algorithm}

The normalized LMS (NLMS) algorithm is a modified form of the standard LMS algorithm. The NLMS algorithm updates the coefficients of an adaptive filter by using the following equation (3) 


$$
\begin{gathered}
\varpi(n+1)=\varpi(n)+\mu \cdot e(n) \cdot{\frac{\bar{u}(n)}{\|\bar{u}(n)\|^{2}}}^{-----(3)} \\
\text { Where } \mu(n)=\mu /\|\bar{u}(n)\|^{2} \text {----- (4) }
\end{gathered}
$$

The NLMS algorithm works same as the standard LMS algorithm except that it uses time-varying step size $\mu(n)$. The advantage of this varying step size will improve the convergence rate but the strength of the signal is still maintained. In contrast to LMS algorithm, the error signal is comparatively smaller in NLMS [11]. Also it is observed that the convergence rate of the NLMS algorithm is greater than that of the standard LMS algorithm because of multiplication operations.

\subsection{Recursive Least Squares algorithm}

(RLS)

The RLS filter is a recursive implementation of the Wiener filter which is used to find the difference between the desired and actual signal. RLS algorithm has the potential to automatically adjust the coefficients of a filter, even though the statistics measures of the input signals are not presented. This algorithm performs at each instant an exact minimization of the sum of the squares of the desired signal estimation errors [6]. Since it utilizes all the information contained in the input data, the estimation is updated recursively when the arrival of new sample. The design of RLS algorithm is given in Figure 3.

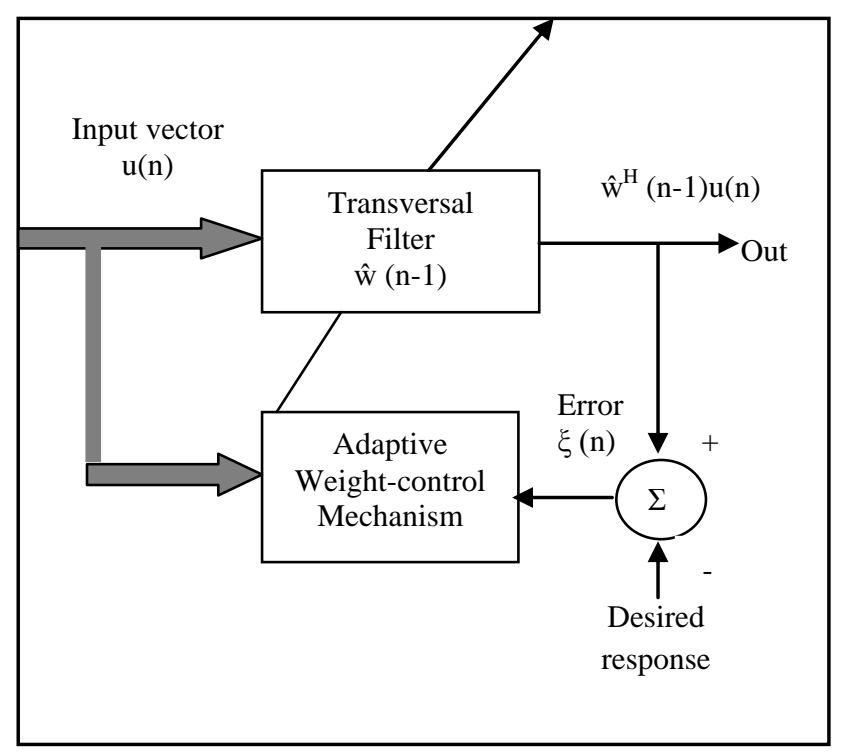

Figure 3: Design of RLS algorithm

The steps involved in RLS algorithm are given bellow.
Steps in RLS algorithm

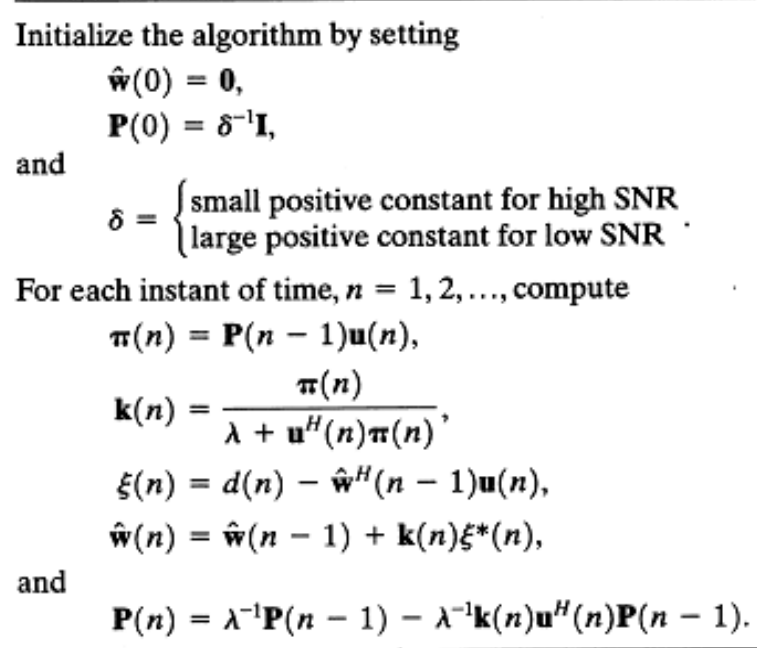

Compared to the LMS algorithm, the RLS approach offers faster convergence and smaller error with respect to the unknown system, at the expense of requiring more computations.

\section{EXPERIMENTAL RESULTS}

LMS and NLMS algorithms have a step size that determines the amount of correction applied as the filter adapts from one iteration to the next. Choosing the appropriate step size plays a major role in adaptive filter design.

- A step size that is too small increases the time for the filter to converge on a set of coefficients. This becomes an issue of speed and accuracy.

- A step size that is too large may cause the adapting filter to diverge, never reaching convergence. In this case the resulting filter might not be stable.

Based on the above criteria, it was found from the experiments that the results highly depend on the step size value. Various step values are tested with the different datasets and it is clear from the experiments that the smaller step sizes improve the accuracy of the convergence of the filter to match the characteristics of the unknown to adapt. It is also observed that a larger step size gives a faster response, but if it is too large, then the result is not satisfactory [10]. In this paper, 0.2 was found to be the optimal step size for the LMS.

For experiments, the separate noise corpus from NOIZEUS were collected and added to the continuous Tamil Speech signals. The performances of these algorithms are investigated for speech enhancement in different noise conditions. Totally 15 datasets were generated for this research work. Three types of noises were implemented namely white noise, pink noise and babble noise at 5, 10,-5 and $-10 \mathrm{~dB}$ SNR. The following figure 4 shows the speech signal noise cancellation for white noise at $10 \mathrm{~dB}$ using LMS, NLMS and RLS algorithms respectively. 


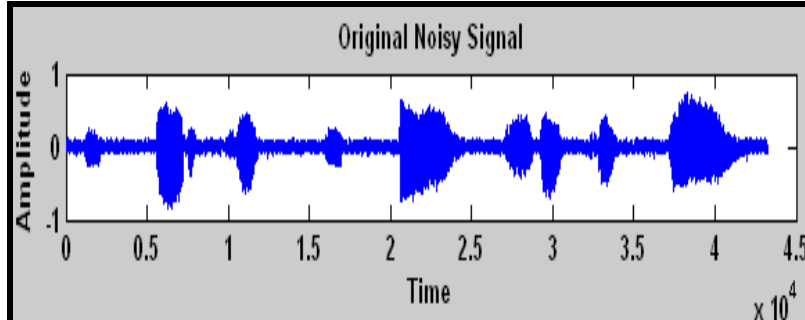

Enhanced Signal using LMS algorithm

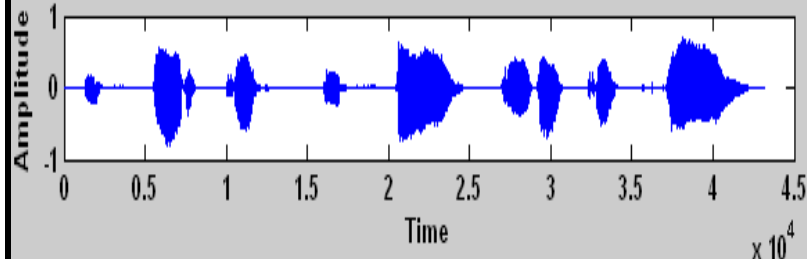

Enhanced Signal using NLLS algorithm

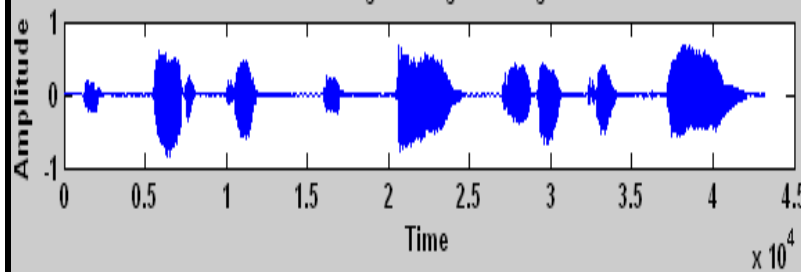

Enhanced Signal using RLS algorithm

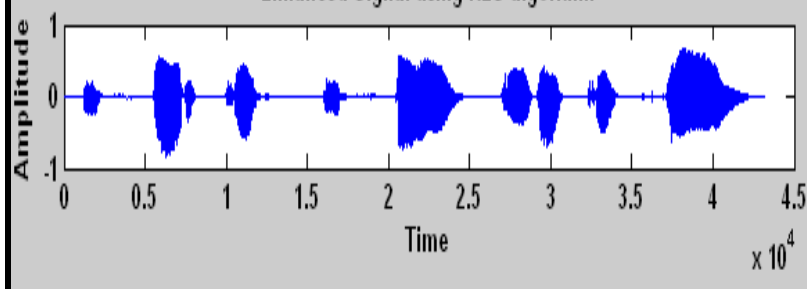

Figure 4: Results of noise cancellation for white noise at 10dB SNR using LMS, NLMS and RLS algorithms

\section{PERFORMANCE EVALUATION}

The primary objective of the adaptive filter is to minimize the error signal $\mathrm{e}(\mathrm{k})$. The success of this minimization will clearly depend on the nature of the input signals and the adaptive algorithm used. The quality of speech signals is a subjective measure which reflects the way the signal is perceived by listeners. At $0 \mathrm{~dB}$ the two signals are of equal strength and negative values are associated with loss of intelligibility due to masking whereas positive values are usually associated with better intelligibility. All the three algorithms obtained a positive and higher SNR values. The performances of these algorithms are measured based on the metrics namely PSNR, MSE, SNR and SNR Loss which are explained below.

\subsection{Mean Squared Error (MSE)}

The Mean Squared Error (MSE) of an estimator is used to quantify the difference between values implied and the true values being estimated. It is calculated using the formula

$$
M S E=\frac{\sum\left(y_{i}-\hat{y}_{i}\right)}{n-p}
$$

\subsection{Peak Signal to Noise Ratio (PSNR)}

The Peak Signal-to-Noise Ratio (PSNR) is the ratio between a signal's maximum power and the power of the signal's noise. It is calculated by the formula (6)

$$
P S N R=10 \log _{10}\left[\frac{R^{2}}{M S E}\right]
$$

\subsection{Signal-to-Noise Ratio (SNR)}

SNR is defined as the ratio of power between the signal and the unwanted noise. One of the most important goals of any speech enhancement technique is to achieve the highest possible SNR. The higher the ratio the better the performance in noise cancellation or reduction. SNR is calculated using the formula (7)

$$
\frac{S}{N}=\frac{n_{\text {signal }}}{n_{\text {noise }}}
$$

Where $n_{\text {signal }}$ the original is signal and $n_{\text {noise }}$ is the noisy signal.

\subsection{SNR loss:}

SNR loss is the increased signal-to-noise ratio required by an individual to understand speech in noise, as compared to normal performance. It is a new objective measure for predicting the intelligibility of noise-suppressed speech [5]. It provides an efficient way to determine the ability of a person to hear speech in background noise. The following table 1 illustrates the range of SNR Loss score and its remarks.

Table 1. Range of SNR Loss Score and its Remarks

\begin{tabular}{|l|l|l|}
\hline $\begin{array}{l}\text { SNR } \\
\text { loss }\end{array}$ & $\begin{array}{l}\text { Degree of } \\
\text { SNR loss }\end{array}$ & $\begin{array}{l}\text { Expected improvement with } \\
\text { directional microphones }\end{array}$ \\
\hline $0-3 \mathrm{~dB}$ & $\begin{array}{l}\text { Normal/near } \\
\text { normal }\end{array}$ & $\begin{array}{l}\text { May hear better than normal } \\
\text { hear in noise }\end{array}$ \\
\hline $3-7 \mathrm{~dB}$ & Mild SNR loss & $\begin{array}{l}\text { May hear almost as well as } \\
\text { normal hear in noise }\end{array}$ \\
\hline $\begin{array}{l}7-15 \\
\mathrm{~dB}\end{array}$ & $\begin{array}{l}\text { Moderate SNR } \\
\text { loss }\end{array}$ & $\begin{array}{l}\text { Moderate SNR loss } \\
\text { Directional microphones help. } \\
\text { Consider array microphones }\end{array}$ \\
\hline
\end{tabular}




\begin{tabular}{|l|ll|l|}
\hline$>15 \mathrm{~dB}$ & $\begin{array}{l}\text { Severe } \\
\text { loss }\end{array}$ & SNR & $\begin{array}{l}\text { Maximum SNR improvement } \\
\text { is needed. Consider FM } \\
\text { system }\end{array}$ \\
\hline
\end{tabular}

The following figures $5,6,7$ and 8 shows the average performance evaluation for the 15 datasets using LMS, NLMS and RLS algorithms for $10 \mathrm{~dB}$ white noise based on MSE, PSNR, SNR and SNR loss respectively.

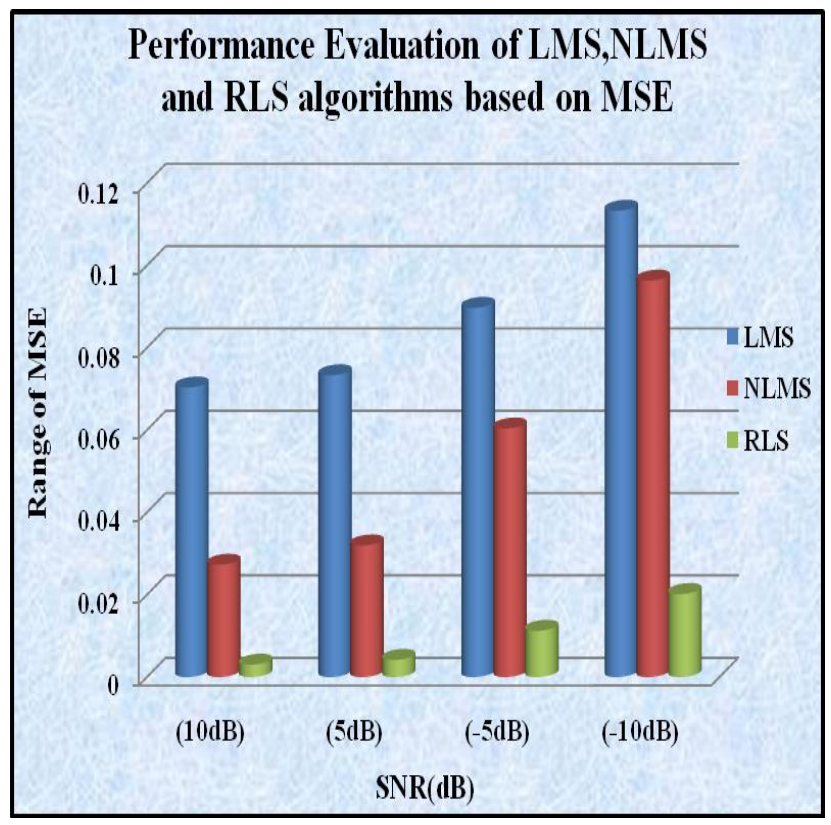

Figure 5: Performance Evaluation of LMS, NMLS and RLS based on MSE

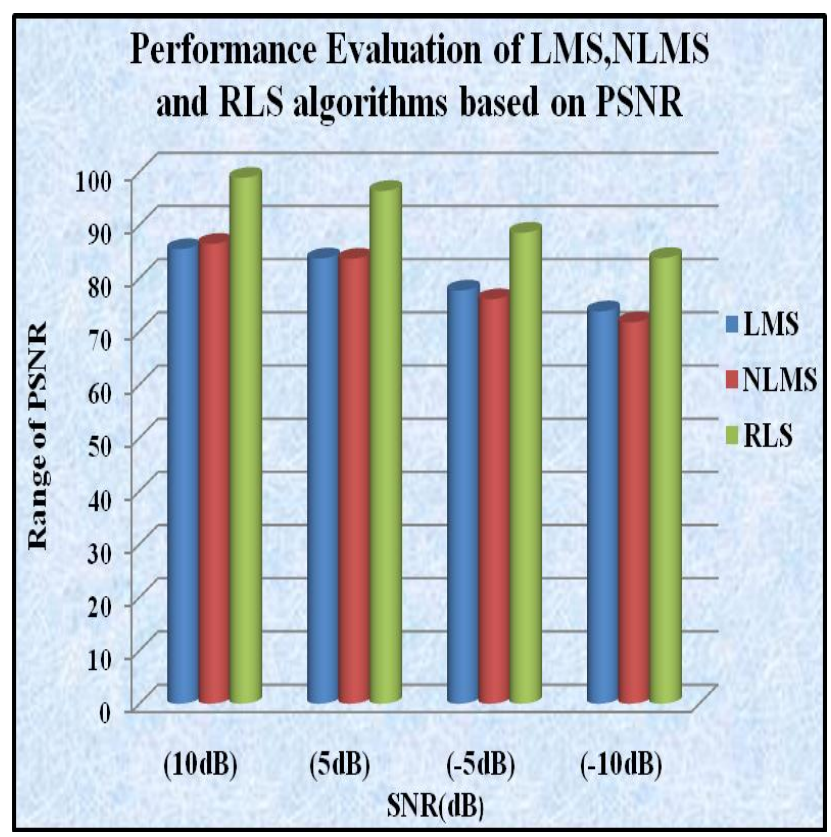

Figure 6: Performance Evaluation of LMS, NMLS and RLS based on PSNR

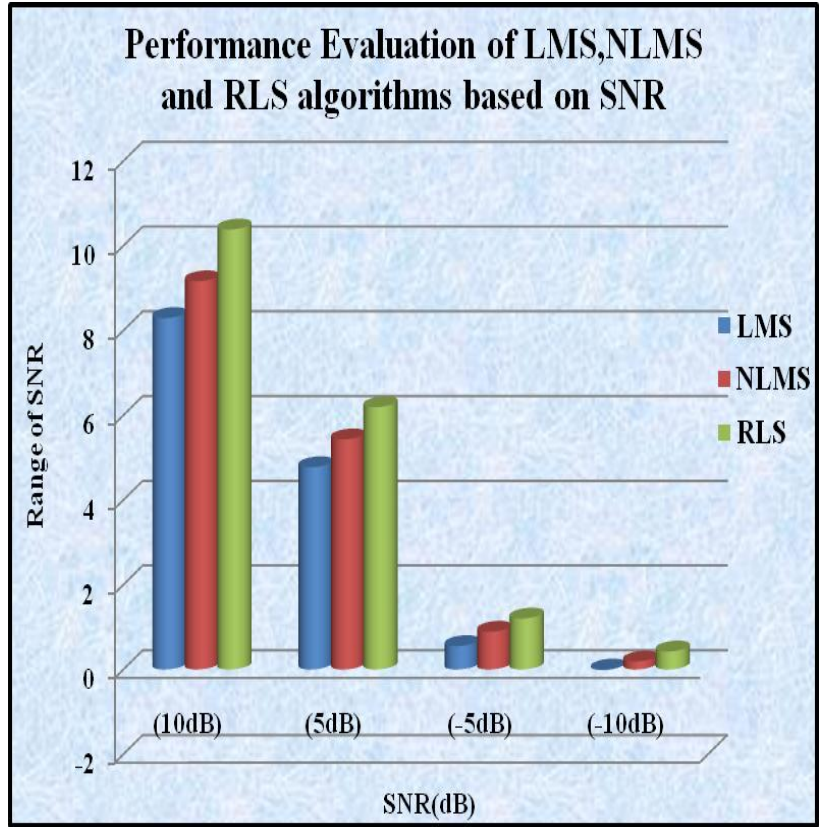

Figure 7: Performance Evaluation of LMS, NMLS and RLS based on SNR

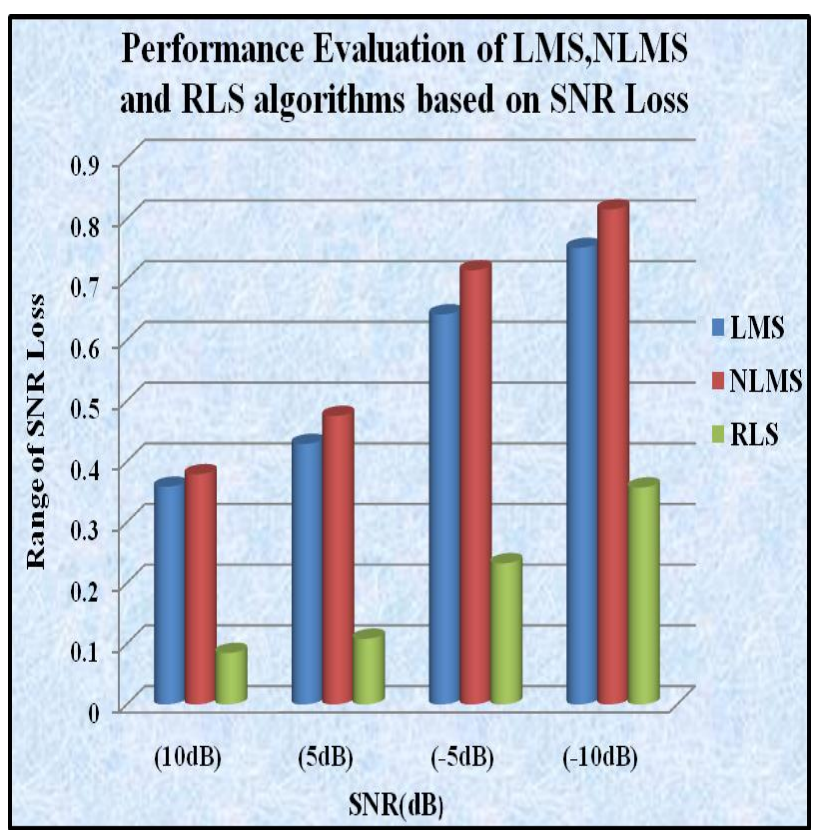

Figure 8: Performance Evaluation of LMS, NMLS and RLS based on SNR Loss

It is clear from the above figures that the RLS algorithm offers least MSE and SNR loss values and it also achieves the highest SNR and PSNR values for all types of noise at different SNR level. All the above algorithms obtained the SNR loss score of the range between $0-3 \mathrm{~dB}$ generally and $0-1 \mathrm{~dB}$ for this particular experiment. The following table 2 shows the comparison of the adopted algorithms based on the average value of the 15 datasets. 
Table 2. Comparison of LMS, NLMS and RLS algorithms for White Noise, Pink Noise and Babble Noise based on MSE, PSNR, SNR and SNR Loss Values

\begin{tabular}{|c|c|c|c|c|c|c|c|c|c|c|c|c|c|}
\hline \multirow{2}{*}{$\begin{array}{l}\text { Type of } \\
\text { Noise }\end{array}$} & \multirow{2}{*}{$\begin{array}{l}\text { SNR } \\
\text { (dB) }\end{array}$} & \multicolumn{4}{|c|}{ LMS } & \multicolumn{4}{|c|}{ NLMS } & \multicolumn{4}{|c|}{ RLS } \\
\hline & & MSE & PSNR & SNR & $\begin{array}{l}\text { SNR } \\
\text { Loss }\end{array}$ & MSE & PSNR & SNR & $\begin{array}{l}\text { SNR } \\
\text { Loss }\end{array}$ & MSE & PSNR & SNR & $\begin{array}{l}\text { SNR } \\
\text { Loss }\end{array}$ \\
\hline \multirow{4}{*}{$\begin{array}{l}\text { White } \\
\text { Noise }\end{array}$} & $10 \mathrm{~dB}$ & 0.07037 & 85.4591 & 8.29484 & 0.35858 & 0.02729 & 86.4491 & 9.16253 & 0.37919 & 0.003015 & 98.8535 & 10.3839 & 0.08469 \\
\hline & $5 \mathrm{~dB}$ & 0.07333 & 83.7014 & 4.77466 & 0.42913 & 0.03198 & 83.6406 & 5.43058 & 0.47517 & 0.004185 & 96.4027 & 6.19259 & 0.10811 \\
\hline & $-5 \mathrm{~dB}$ & 0.08972 & 77.6766 & 0.55280 & 0.64254 & 0.06040 & 76.0563 & 0.89055 & 0.71525 & 0.011258 & 88.5507 & 1.20227 & 0.23246 \\
\hline & $-10 \mathrm{~dB}$ & 0.11330 & 73.7633 & -0.0138 & 0.75208 & 0.09632 & 71.7056 & 0.18780 & 0.81557 & 0.020185 & 83.7926 & 0.42496 & 0.35728 \\
\hline \multirow{4}{*}{$\begin{array}{l}\text { Pink } \\
\text { Noise }\end{array}$} & $10 \mathrm{~dB}$ & 0.07096 & 84.8721 & 8.40980 & 0.37176 & 0.02840 & 85.6988 & 9.33851 & 0.39491 & 0.003105 & 98.7224 & 10.3749 & 0.08682 \\
\hline & $5 \mathrm{~dB}$ & 0.07509 & 82.6522 & 4.88625 & 0.44901 & 0.03512 & 82.5266 & 5.61283 & 0.49519 & 0.004401 & 96.0796 & 6.19492 & 0.10781 \\
\hline & $-5 \mathrm{~dB}$ & 0.09952 & 75.8056 & 0.66277 & 0.65780 & 0.06939 & 74.3824 & 1.07284 & 0.72209 & 0.012135 & 87.9476 & 1.20365 & 0.21413 \\
\hline & $-10 \mathrm{~dB}$ & 0.12756 & 71.6294 & 0.09826 & 0.75632 & 0.11064 & 69.9061 & 0.37208 & 0.81447 & 0.021877 & 83.1015 & 0.42630 & 0.33618 \\
\hline \multirow{4}{*}{$\begin{array}{l}\text { Babble } \\
\text { Noise }\end{array}$} & $10 \mathrm{~dB}$ & 0.07310 & 83.7498 & 8.66351 & 0.36906 & 0.03047 & 84.2702 & 9.83596 & 0.38221 & 0.003308 & 98.1071 & 10.3908 & 0.09134 \\
\hline & $5 \mathrm{~dB}$ & 0.07924 & 81.0092 & 5.15612 & 0.44218 & 0.0394 & 80.6938 & 6.13017 & 0.47200 & 0.004822 & 95.1550 & 6.19897 & 0.11112 \\
\hline & $-5 \mathrm{~dB}$ & 0.11336 & 73.4019 & 0.93806 & 0.62575 & 0.08640 & 72.0793 & 1.60382 & 0.67210 & 0.013582 & 86.6958 & 1.20983 & 0.21261 \\
\hline & $-10 \mathrm{~dB}$ & 0.15100 & 69.1012 & 0.36503 & 0.71630 & 0.13656 & 63.3543 & 0.89245 & 0.76273 & 0.024582 & 81.7553 & 0.43357 & 0.33283 \\
\hline
\end{tabular}

\section{CONCLUSION}

The performance of speech communication system is degraded when the input signal contains a significant level of noise. As a result, speech quality, speech intelligibility, or recognition rate requirements cannot be met. Improvements are obtained when the speech processing system is combined with a speech enhancement preprocessor. In this paper, the three widely used adaptive filters such as LMS, NLMS and RLS algorithms have accomplished for Tamil speech noise cancellation. Among these, LMS algorithm is a very simple and effective method to implement though it is a slower one. Even though, with increased step size, the rate of convergence obtained in NLMS is not up to the satisfactory level. The experimental results show that the RLS algorithm makes the converging speed and also provides better noise reduction and improved speech quality and intelligibility when compared to the other algorithms. As a result, with these appropriate settings of the adaptive filter parameters, this optimal signal can be employed for the speech recognition system as a front end.

\section{REFERENCES}

[1] Georgi Iliev and Nikola Kasabov, "Adaptive Filtering with Averaging in Noise Cancellation for Voice and Speech Recognition", Department of Information Science, University of Otago.
[2] V.JaganNaveen, T.prabakar, J.Venkata Suman , P.Devi Pradeep, "Noise suppression in speech signals using adaptive algorithms", International Journal of Signal Processing, Image Processing and Pattern Recognition,Vol. 3, No. 3, September, 2010.

[3] Jan Vanus and Vitezslav Stkskala,"Application of optimal settings of the LMS adaptive filter for speech signal prosessing", Procedings of the International multiconference on Computer Science and Information Technology pp.767-774, ISBN 978-83-60810-27-9,ISSN 1896-7094.

[4] Jan Vanus, "The use of the adaptive noise cancellation for voice Communication with the control system", International Journal of Computer Science and Applications,Technomathematics Research Foundation,Vol. 8, No. 1, pp. 54 - 70, 2011.

[5] Jianfen Maa b, Philipos C. Loizou b, "SNR loss: A new objective measure for predicting the intelligibility of noise-suppressed speech", J. Ma, P.C. Loizou / Speech Communication 53 (2011) 340-354.

[6] Komal R. Borisagar and Dr. G.R.Kulkarni,"Simulation and Comparative Analysis of LMS and RLS Algorithms Using Real Time Speech Input Signal”, Global Journal of Researches in Engineering, Page 44,Vol.10,Issue,5 (Ver1.0)October2010.

[7] D.Prabhakara Rao,Sri M.Koteswararao, Dr.I.Santhi Prabha, "Combinational Adaptive Filter Technique for Speech Enhancement”, IJECT Vol. 2, SP-1, Dec . 2011, ISSN : 2230-7109(Online) | ISSN : 2230-9543(Print). 
[8] Sayed. A. Hadei, Student Member IEEE and M. lotfizad, "A Family of Adaptive Filter Algorithms in Noise Cancellation for Speech Enhancement", International Journal of Computer and Electrical Engineering, Vol. 2, No. 2, April 2010,1793-8163.

[9] Simon Haykin,"Adaptive Filter Theory", prentice hall of india, $4^{\text {th }}$ Edition.

[10] Siva Prasad Nandyala and T. Kishore Kumar, "Speech Enhancement Using Kernel Adaptive Filtering Method", Microwaves, Communications, Antennas and Electronics Systems (COMCAS), 2011 IEEE International
Conference on, 10.1109/COMCAS.2011.6105874 , ISBN: 978-1-4577-1692-8.

[11] A. Srinivasan, "Adaptive Echo Noise Elimination for Speech Enhancement of Tamil letter 'Zha'", International Journal of Engineering and Technology Vol.1 (3), 2009, 91-97, ISSN: 0975-4024.

[12] L. Stasionis, A. Serackis , "Selection of an Optimal Adaptive Filter for Speech Signal Noise Cancellation using C6455 DSP”, Electronics And Electrical Engineering, ISSN 1392 - 1215, 2011. No. 9(115). 